\title{
A method combining TA cloning and fluorescence screening for rapid acquisition of transgenic seeds
}

\author{
Min Li ${ }^{\ddagger}$, , Ling-Yan Jiang ${ }^{\ddagger 1}$, Qin Liu ${ }^{1,3}$, Yuan-Hang Wu' ${ }^{1}$ Guo-Dao Liu' ${ }^{2}$, Yin-Hua Chen' \& Li-Juan Luo*,1
}

\section{ABSTRACT}

The establishment of transgenic plants has greatly promoted the progress of plant research. However, traditional selection methods using antibiotics or herbicides may miss any positive transformants with growth defects. Additionally, screening with antibiotics/herbicides requires a huge amount of seeds, sterile work conditions and a large amount of space to germinate plants, making the selection process time- and labor-consuming. In this study, we constructed a novel stable transformation vector, plasmid of OLE1-GFP T-DNA vector (pOGT), which can shorten the steps of cloning foreign genes into expression vectors by using TA cloning. Additionally, selection of transformed seeds with fluorescence overcomes the difficulties of conventional selection with antibiotics/herbicides and simplifies the screening process for transgenic plants.

\section{METHOD SUMMARY}

We constructed a stable transformation vector, plasmid of OLE1-GFP T-DNA vector (pOGT) with an OLE1-GFP fusion gene driven by the OLE1 promoter (ProOLE1) as a selection marker. The OLE1 promoter controls the expression of an OLE1-GFP fusion protein and makes the transformed seeds fluorescent. Digestion of pOGT with $X c m l$ generates a single thymidine $(T)$ overhang at the $3^{\prime}$ end which enables the introduction of foreign genes into POGT through one-step TA cloning. We generated transgenic Arabidopsis lines transformed with the POGT-exogenous gene and screened transformed seeds using fluorescence.

\section{KEYWORDS}

Arabidopsis thaliana $\cdot$ OLE1 gene $\cdot$ green fluorescent protein (GFP) • selection marker • stable expression vector $\cdot$ TA cloning

${ }^{1}$ Hainan Key Laboratory for Sustainable Utilization of Tropical Bioresources, College of Tropical Crops, Hainan University, Haikou 570228, China; ${ }^{2}$ Tropical Crops Genetic Resources Institute, Chinese Academy of Tropical Agricultural Sciences, Haikou China; ${ }^{3}$ College of Biology \& Pharmacy, Yulin Normal University, Yulin 537000, China; *Author for correspondence: Tel.: +86 0898 66256127; 990908@hainanu.edu.cn; ₹Co-first authors

BioTechniques 68: 251-256 (May 2020) 10.2144/ btn-2019-0141
Generation of transgenic plants, especially for the model plant Arabidopsis thaliana, is essential for characterization of gene functions and investigation of molecular mechanisms [1,2]. Identification of the transgenic plants is conventionally achieved with a selection marker, which allows transformed plants to grow on agar plates with an antibiotic or herbicide. However, such traditional screening methods have several major drawbacks. First, the expression of some genes may cause developmental growth defects in the transformants, and conventional selection with antibiotic or herbicide may miss such transformed plants [3]. Second, screening for transformants able to grow in the presence of antibiotics/ herbicides requires sterile work conditions, a large amount of seeds and space to grow the plants. The development of a system that overcomes these difficulties could greatly facilitate the screening of transgenic plants.

OLE1 (GenBank accession name: At4G25140) is a type of oleosin, which are themselves structural proteins embedded in the membranes surrounding the oil bodies [4]. Studies have shown that OLE1 is specifically localized on the oil body membrane in the seed cells and can be used as a screening marker for Arabidopsis [5]. Therefore, a fusion gene with an OLE1 promoter driving a fluorescent reporter (ProOLE1::OLE1-GFP) may be used as an endogenous selection marker to identify transgenic Arabidopsis at the seed stage. The pCXSN vector is a commercial plant-stable expression vector, which contains a $\operatorname{ccd} B$ lethal gene. Digestion of the $\mathrm{pCXSN}$ vector with $X \mathrm{~cm} /$ removes $c c d B$ and generates a 3 '-T overhang, allowing the introduction of foreign genes using TA cloning [6].
In this study, we report a novel technique which combines pCXSN plant expression vector as vector backbone with Pro $_{\text {OLE }}:$ OLE1-GFP as screening marker. The recombinant vector is named pOGT (plasmid of OLE1-GFP T-DNA vector). pOGT could be used as T-DNA vectors which have 3 '-T overhangs after restriction endonuclease digestion. Therefore, TA cloning could be performed for cloning of PCR-amplified fragments into the pOGT vector. The resulting construct could be introduced into Arabidopsis, and the transformed seeds could be screened by fluorescence.

\section{MATERIALS \& METHODS Plant materials \& growth}

Arabidopsis thaliana seeds (Col-0) were sterilized with $70 \%$ ethanol and incubated at $4^{\circ} \mathrm{C}$ for $2-3$ days to break seed dormancy, then sown on nutrient soil for 1 week. The seedlings were transferred to $10-\mathrm{cm}^{2}$ pots at a density of four plants/pot for growth. Arabidopsis plants were grown at $22^{\circ} \mathrm{C}$ under long-daylight conditions (light 9-12 h/ day) with sufficient fertilizers to obtain a large number of flowers. The seeds were provided by Hainan Key Laboratory for Sustainable Utilization of Tropical Bioresources.

\section{Plasmid DNA construction of the pOGT vector}

The forward and reverse primers pOLE1-F and pOLE1-F (Table 1) were designed based on the Arabidopsis PrO $_{\text {OLE }}$ ::OLE1 sequence from The Arabidopsis Information Resource website [7]. Extraction of Arabidopsis genomic DNA was performed according to the cetrimonium bromide (CTAB) method [8]. The PrO ${ }_{\text {OLE }}:$ OLE1 gene was amplified by PCR using the Arabidopsis genomic DNA and specific primers pOLE1-F and pOLE1-R. The 


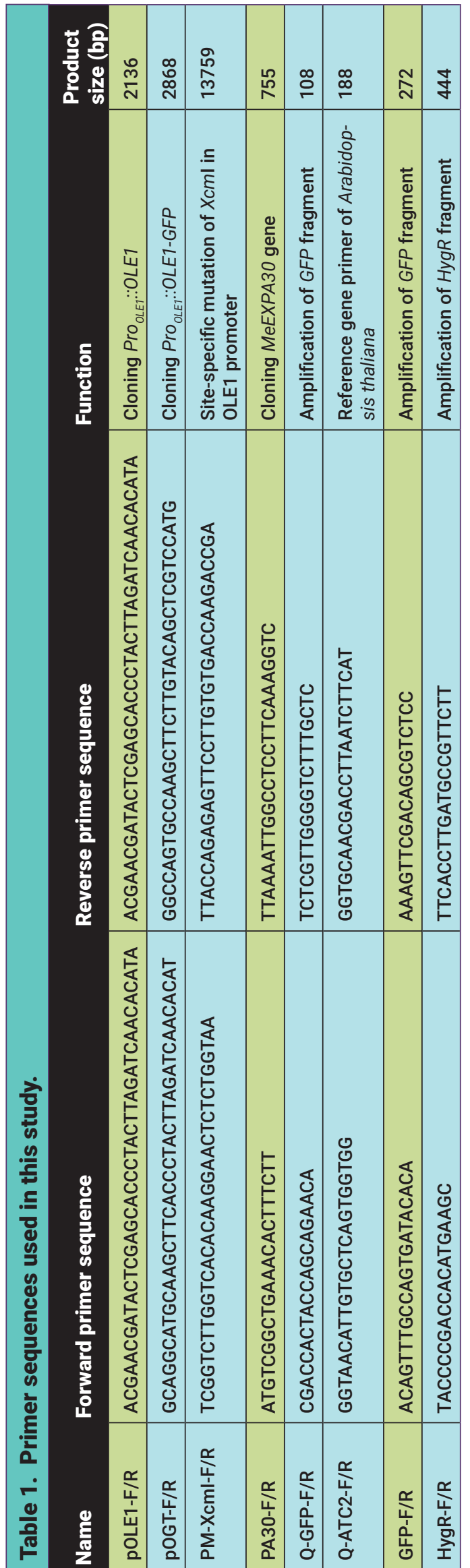

pA7-GFP vector (BioVector) was digested with Xhol and Sall (New England Biolabs), and the resulting 4591-bp fragment was purified by gel recovery using the Omega gel extraction kit following the manufacturer's instructions. Next, the Pro ${ }_{\text {OLEE }}:$ OLE1 fragment and pA7-GFP fragment were linked by homologous recombination using Vazyme ClonExpress II one-step cloning kit.

The primers POGT-F and POGT-R were designed according to the sequence of Pro $_{\text {OLE }}::$ OLE1-GFP (Table 1). The Pro ${ }_{\text {OLE }}::$ OLE1GFP gene was amplified by PCR using the pA7-OLE1-GFP plasmid as a template and the corresponding primers. The pCXSN vector [6] was digested with HindIII (New England Biolabs), the 10,802-bp fragment was purified by gel recovery (Omega). After that, the Pro ${ }_{\text {OLEY }}:$ OLE 1-GFP fragment and digested PCXSN fragment were linked by homologous recombination (Vazyme), generating PCXSN-OLE1-GFP.

The $X \mathrm{CmI}$ recognition site of the OLE1 promoter on the PCXSN-OLE1-GFP vector was eliminated by site-specific mutation, using the primers (PM-Xcml-F, PM-Xcml-R) listed in Table 1, according to the method of the QuickMutation $^{\text {TM }}$ Site-Directed Mutagenesis Kit (Beyotime). The complete sequence of POGT was deposited in NCBI with the accession number Banklt2292911 pOGT MN832866.

\section{Plasmid DNA construction of pOGT- MeEXPA30 vector}

MeEXPA30 (Manes.S055800) was amplified by PCR using DNA polymerase (Takara primeSTAR GXL DNA polymerase) and primers (PA30-F, P30-R) listed in Table 1. The $A$-addition procedure for the amplified product was performed using the Takara DNA A-Tailing Kit. The plant expression vector POGT was digested with $X \mathrm{cml}$ restriction enzyme (New England Biolabs) to obtain a T-DNA vector. The digested POGT plasmids and the MeEXPA30 were purified from agarose gels after electrophoresis using the Omega gel extraction kit and ligated with T4 DNA ligases (Thermo). The ligation reaction was carried out in a total volume of $20 \mu \mathrm{l}$ containing $60 \mathrm{ng}$ of T-DNA vector and the corresponding volume of PCR product with an insert-to-vector molar ratio of around 3:1. The ligation reaction mixture was transformed into Escherichia coli strain Trans1-T1 (TransGen Biotech, Beijing, China) by heat-shock transformation $\left(42^{\circ} \mathrm{C}, 30 \mathrm{~s}\right)$. 
All recombinant plasmids identified from positive colonies were verified by sequencing.

\section{Transformation of Arabidopsis plants}

The pCXSN-OLE1-GFP, pOGT and pOGTMeEXPA30 plasmids were introduced into Agrobacterium tumefaciens GV3101 (Weidi Biotechnology, Shanghai, China) by a freezethaw method. Arabidopsis Col-0 plants were transformed via the floral-dip method as described previously [9].

\section{Screening of transgenic Arabidopsis}

The dried seeds of wild-type (Col-0) and transformed Arabidopsis were collected and screened under fluorescence microscope (Leica M205FA/DFC550). Transgenic Arabidopsis seeds ( $T_{1}$ seeds) with green fluorescent protein (GFP+) were grown on soil media (vermiculite:nutrient soil = 1:1), generating $T_{1}$ transgenic plants. Seeds collected from $T_{1}$ plants were $T_{2}$ seeds. For genotyping $T_{1}$ plants, the genomic DNA of $T_{1}$ plants was extracted by the CTAB method [8]. Amplification of Pro $_{O L E T}:$ OLE1GFP, MeEXP30, GFP, HygR and Actin2 in $\mathrm{T}_{1}$ plants was performed using the corresponding primers listed in Table 1.

\section{Quantitative real-time PCR}

For examining the transcript levels of GFP in plants transformed with $\mathrm{POGT}$ and $\mathrm{PCXSN}$ OLE1-GFP, the total RNA was extracted from leaves of 2- to 3-week-old plants with the RNA-plant Plus Kit (Tiangen Biotech, Beijing, China), and reverse transcribed to cDNA using the PrimeScript RT reagent Kit (TaKaRa, Dalian, China) following the product instructions. Levels of GFP mRNA in these two transgenic plants were detected using the SYBR real time PCR Kit (TaKaRa, Dalian, China). Three independent experiments were performed. Expression levels were calculated using the double-standard curves method [10]. ACT2 (GenBank accession name:AT3G18780) was used as the reference gene for normalization [11] and the primers for amplification are listed in Table 1. Statistical analyses were performed using two-sample unpaired $t$-tests.

\section{RESULTS \& DISCUSSION}

\section{Construction of pOGT vector}

A schematic illustration of the pOGT construction is shown in Figure 1. The fragment of OLE1 gene with its native

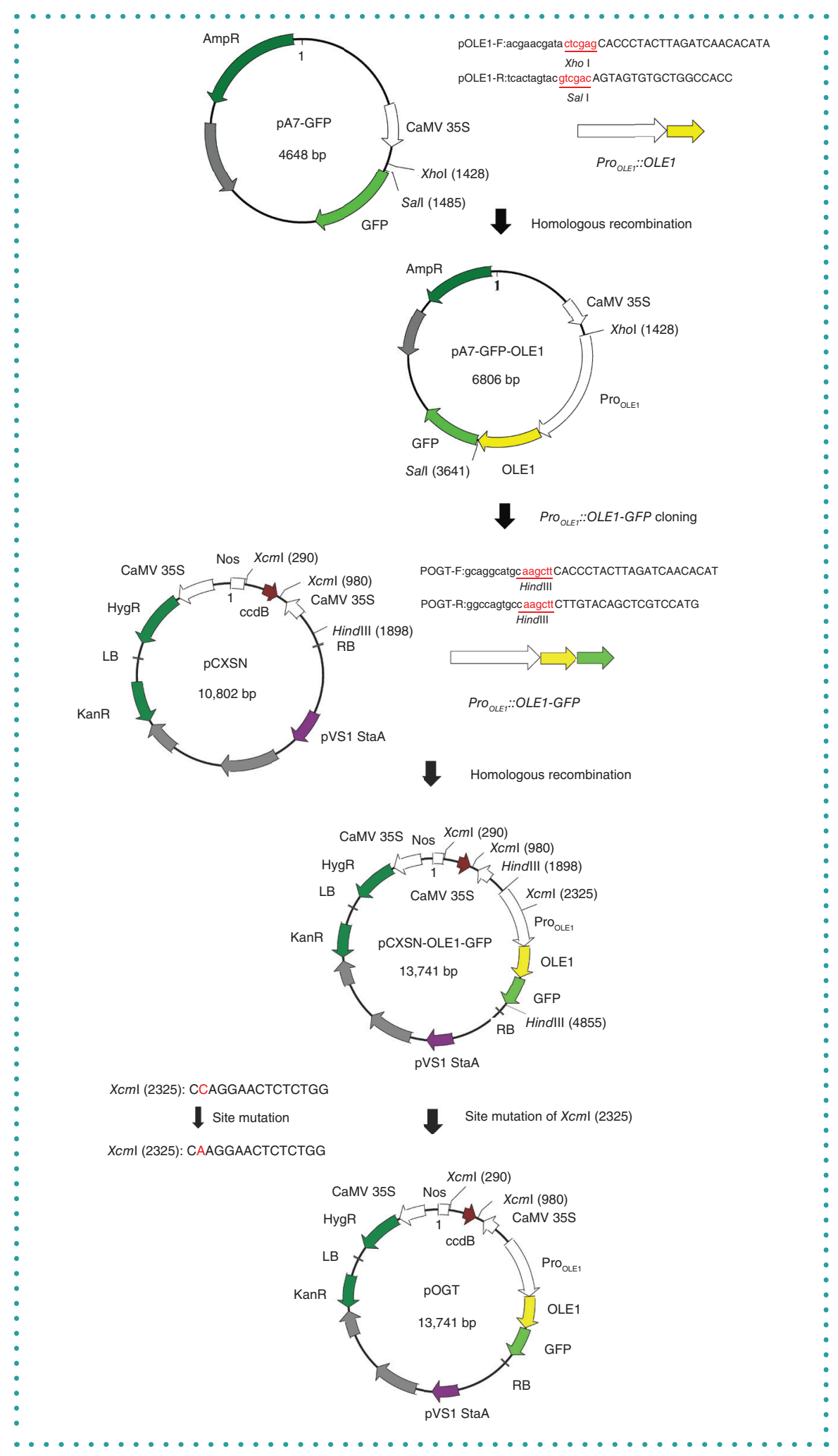

Figure 1. Schematic illustration of POGT construction. The Pro ${ }_{O L E 1}:: O L E 1$ fragment was amplified from the Arabidopsis genomic DNA and cloned into the PA7 vector. The fragment of Pro ${ }_{\text {OLEF }}:$ OLE1GFP was amplified from the recombinant pA7 vector and cloned into the pCXSN vector. The $X c m$ site in the promoter of OLE1 was mutated, resulting in the final construct of the POGT vector. In the primers, the 16-bp upstream sequence from restriction sites on the vector is represented by lowercase letters, and the restriction sites are represented by red lowercase letters. POGT: Plasmid of OLE1-GFP T-DNA vector; Pro $_{\text {OLEF }}::$ OLE1: OLE1 promoter driving a fluorescent reporter. 


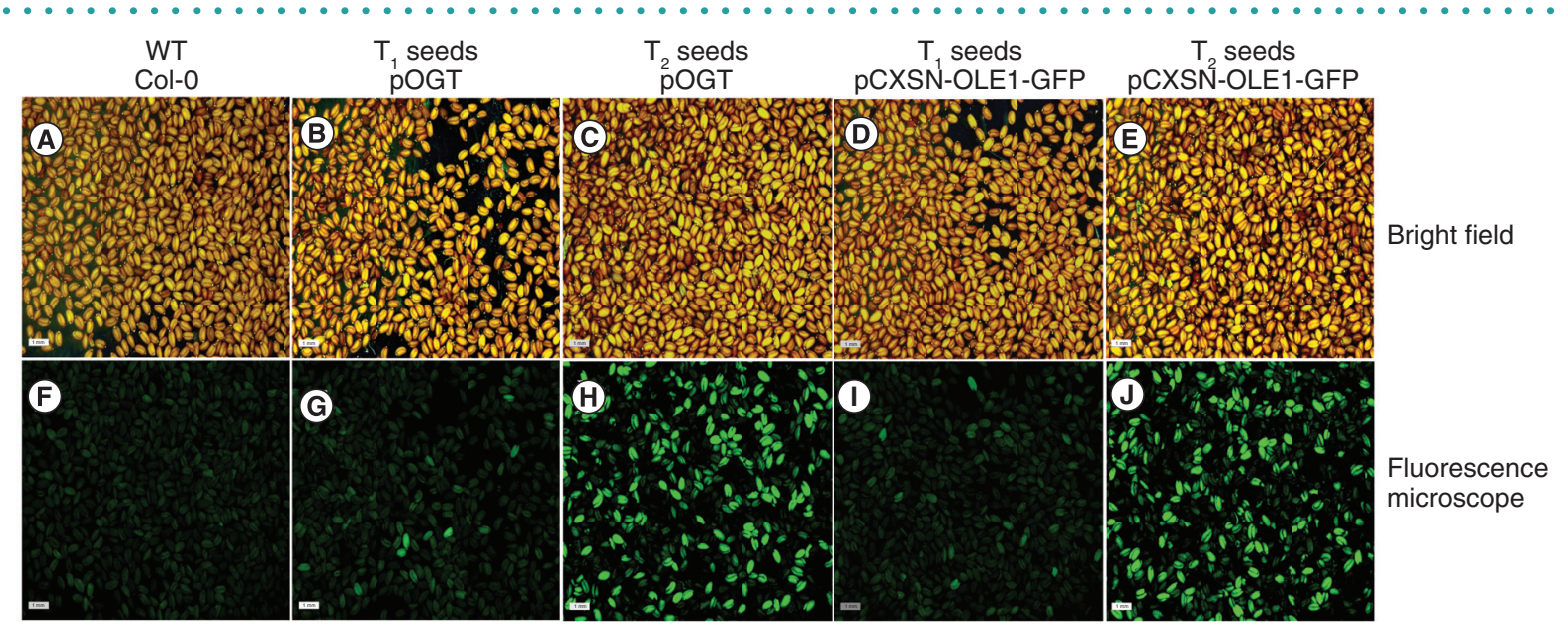

(K)

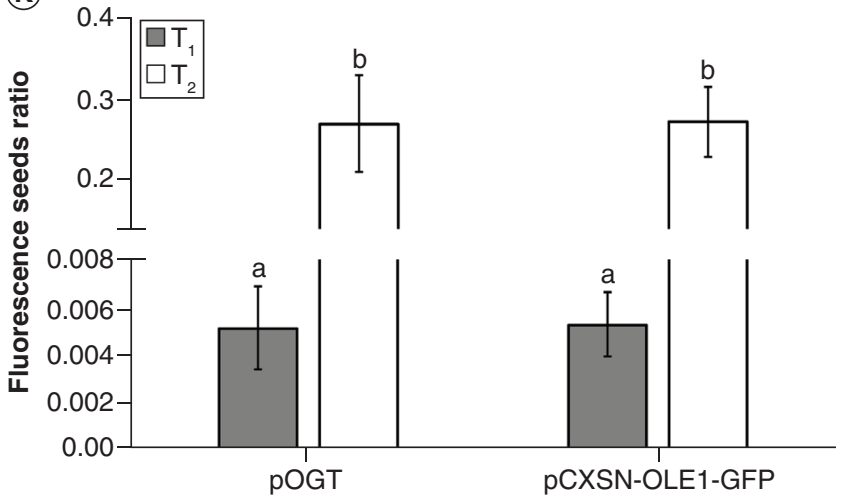

(L)
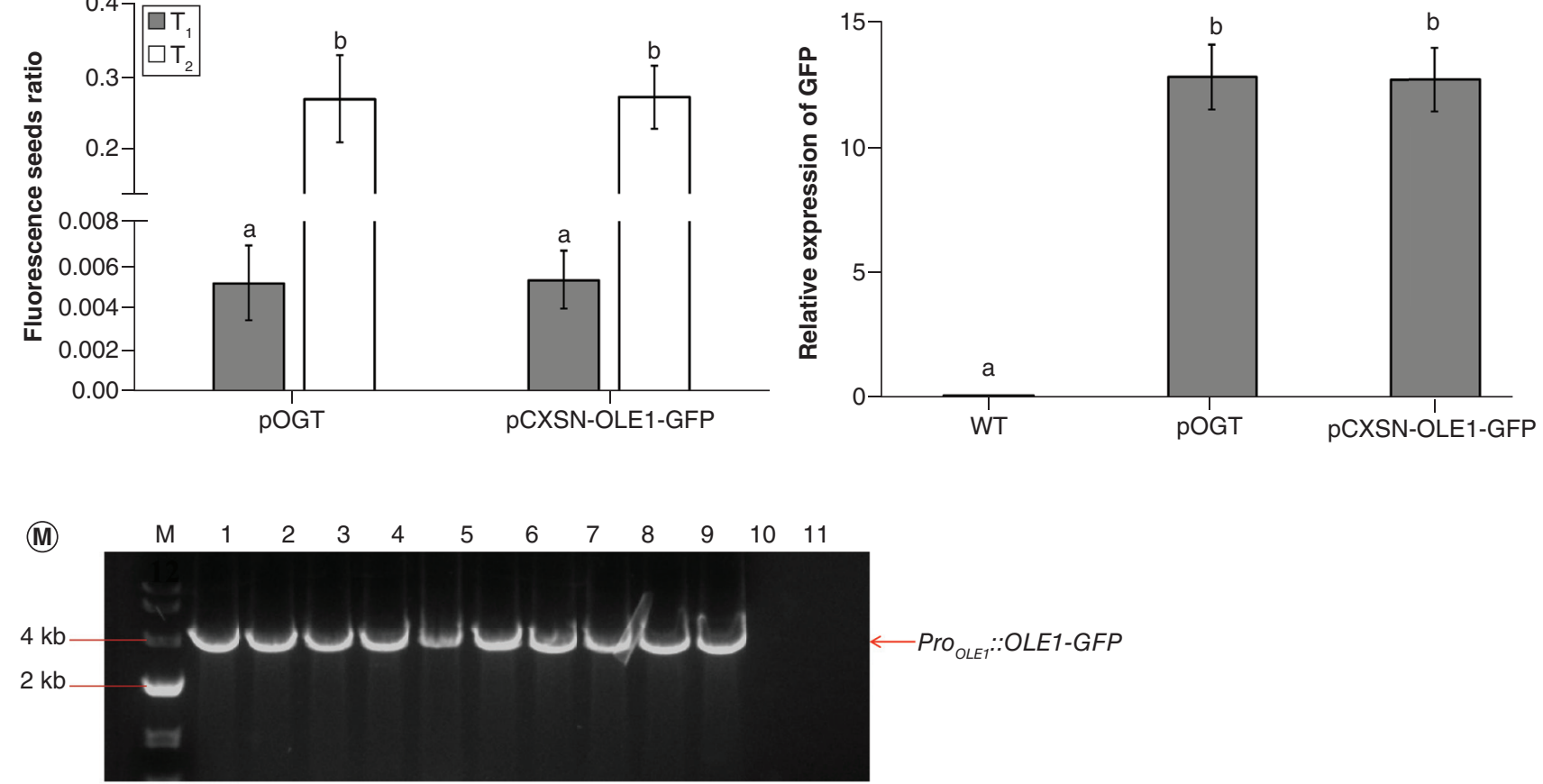

Figure 2. Fluorescence and molecular detection of transgenic Arabidopsis seeds transformed with the pOGT and pCXSN-OLE1-GFP vectors.

(A-E) Seeds viewed under bright-field microscopy. (F-J) The same fields under fluorescence microscope. (A \& F) Col-0 (WT) Arabidopsis seeds.

(B \& G) $T_{1}$ generation seeds of pOGT transgenic Arabidopsis. (C \& H) $T_{2}$ generation seeds of pOGT transgenic Arabidopsis. (D \& I) $T_{1}$ generation seeds of pCXSN-OLE1-GFP transgenic Arabidopsis. (E \& J) T 2 generation seeds of pCXSN-OLE1-GFP transgenic Arabidopsis. (K) Quantification of transformation efficiency represented by the ratio of fluorescent seed versus the total seed number in each random detection view under the microscope. Graphed are means $\pm S E$, representative of three independent replicates $(n=3)$. Lowercase letters indicate significant groupings $(p<0.01)$ using the $t$-test. $T_{2}$ generation seeds were the progeny of the $T_{1}$ seeds with the strongest fluorescence intensity. (L) mRNA levels of GFP measured by quantitative real-time PCR from leaves of 2-3-week-old Col-0 (WT) and transgenic Arabidopsis transformed with pOGT and pCXSN-OLE1-GFP. Transcript levels were normalized to the amount of $A C T 2$ transcripts detected in each sample. Data were pooled from 3 replicates $(n=3)$. Lowercase letters indicate significant groupings $(p<0.01)$ using the $t$-test. (M) PCR identification of $T_{1}$ transgenic plants expressing pOGT. Ten independent primary plants (Lanes $\left.1-10\right)$ were selected for the analysis. Lane 11 shows no amplification of PrO ${ }_{O L E 1}::$ OLE1-GFP gene from Arabidopsis Col-0 (wild-type). Lane 12 shows no amplification of Pro $_{O L E 1}::$ OLE1-GFP gene without template (negative control).

POGT: Plasmid of OLE1-GFP T-DNA vector; Pro $_{\text {OLE1 }}::$ OLE1-GFP: OLE1 promoter driving a fluorescent reporter; WT: Wild type.

promoter was amplified from the Arabidopsis genome by PCR (Figure 1 \& Supplementary Figure 1A). The forward primer contained a 16-bp upstream sequence and the sequence of Xhol restriction sites on the pA7 vector, and the reverse primer contained a $16-b p$ downstream sequence and the sequence of Sall (Figure 1). The amplified PCR product was inserted into the pA7 vector in the position between $\mathrm{Xhol}$ and Sall by homol- ogous recombination (Figure $1 \&$ Supplementary Figure $1 \mathrm{~B} \& \mathrm{C}$ ). The fragment of OLE1 promoter::OLE1-GFP $\left(\right.$ Pro $_{\text {OLEE: }}:$ OLE1-GFP) was amplified from the recombinant pA7 plasmid with primers containing 16-bp upstream/ 

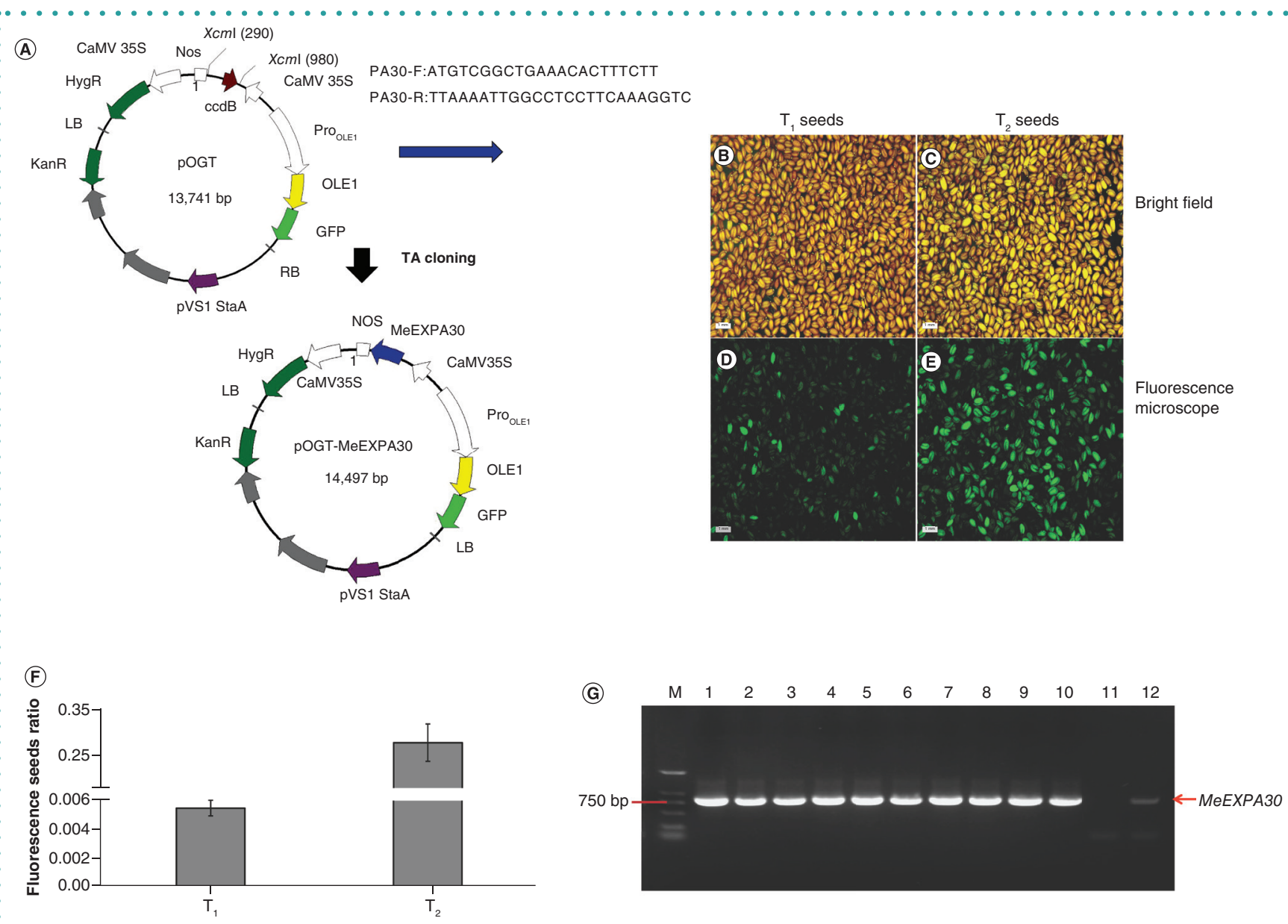

Figure 3. Heterologous expression of cassava MeEXPA30 in Arabidopsis using the pOGT vector. (A) Diagrammatic representation of the pOGT-MeEXPA30 construction. The fragment of MeEXPA30 was amplified from cassava genome and cloned into the pOGT vector through TA cloning. (B \& C) Images from bright-field microscopy. (D \& E) The same fields under fluorescence microscope. (B \& D) $T_{1}$ generation seeds of pOGT-MeEXP30 transgenic Arabidopsis. (C \& E) T 2 generation seeds of pOGT-MeEXP30 transgenic Arabidopsis. (F) Quantification of transformation efficiency represented by the ratio of fluorescent seeds versus the total seed number in each random detection view. Graphed are means $\pm \mathrm{SE}$, representative of three independent replicates $(n=3)$. (G) PCR identification of $T_{1}$ transgenic plants expressing pOGT-MeEXPA30. Ten independent primary plants (Lanes 1-10) were selected for the analysis. Lane 11 shows no amplification of MeEXPA30 from Arabidopsis Col-0 (wild-type). Lane 12 shows the amplification of MeEXPA30 from POGT-MeEXPA30 plasmid. POGT: Plasmid of OLE1-GFP T-DNA vector.

downstream sequences from the HindIII site, and introduced into the stable transformation vector pCXSN, generating the construct of pCXSN-OLE1-GFP (Figure 1 \& Supplementary Figure 1D-F). Because the promoter of OLE1 contains an $X c m l$ recognition site, which would affect the vector used as $X \mathrm{cml}$ digestion-generated T-DNA vector, we mutated the $X c m l$ site by site-directed mutagenesis (Figure 1A \& H). Xcml digestion of the vector with the mutated promoter (pOGT vector) generated two bands, whereas digestion of the construct with the original promoter (pCXSN-OLE1-GFP) resulted in three bands, confirming the elimination of the $X \mathrm{cml}$ site in the OLE1 promoter (Supplementary Figure 1G). Thus we generated the final construct of the POGT vector.

\section{Generation of Arabidopsis transgenic} lines transformed with POGT vector

To investigate the feasibility of generating transgenic lines transformed with the POGT vector, we introduced the vector into Arabidopsis via A. tumefaciens-mediated floral-dip method. The fluorescence of $T_{1}$ seeds was inspected under the fluorescence microscope (Figure 2B \& G). Only $0.5 \%$ of the $T_{1}$ seeds exhibited green fluorescence (Figure $2 B$ \& $G$ \& $K)$. $T_{1}$ seeds with strong green fluorescence were chosen for the propagation of next generation $\left(T_{2}\right)$ seeds. Compared with $T_{1}$ seeds, the proportion of fluorescent seeds in the $T_{2}$ generation increased to $26 \%$ (Figure $2 \mathrm{C} \& \mathrm{H} \& \mathrm{~K}$ ). To examine the introduction of PrO $_{O L E}::$ OLE1-GFP, ten independent $T_{1}$ plants were selected for PCR analysis using their genomic DNA. Amplifications of the full length of PrO $_{\text {OLE }}::$ OLE1-GFP were detected in all ten plants, confirming the results of fluorescence detection (Figure $2 \mathrm{M}$ ).

Because we made the mutation in the OLE1 promoter within the POGT vector, we also transformed the construct with the original promoter ( $\mathrm{pCXSN-OLE1-GFP)} \mathrm{to} \mathrm{compare}$ the effect of the mutation on the transformation efficiency and the expression activity. Fluorescence detection showed that seeds transformed with $\mathrm{POGT}$ vector and $\mathrm{PCXSN}$ OLE1-GFP had similar rates of fluorescent 
seeds in both $\mathrm{T}_{1}$ and $\mathrm{T}_{2}$ seeds (Figure $2 \mathrm{~A}-\mathrm{K}$ ). To examine the expression activity of the mutated OLE1 promoter, we compared the expression level of GFP in $\mathrm{T}_{1}$ plants expressing pOGT versus PCXSN-OLE1-GFP. The results of qPCR showed that the transcript levels of GFP were similar in the transgenic lines transformed with these two constructs (Figure 2L). These results indicate that the mutation does not affect the transformation efficiency or activity of the OLE1 promoter.

To compare the effectiveness of fluorescent screening versus hygromycin selection, 20 independent $T_{1}$ plants were selected for PCR analysis using their genomic DNA. Amplifications of a fragment of GFP, HgyR, were detected in all 20 plants (Supplementary Figure 2), confirming the equal effectiveness of these two methods.

\section{Heterologous expression of cassava} MeEXPA30 in Arabidopsis using pOGT vector

To investigate the utilization of pOGT vector to introduce foreign genes into Arabidopsis, we generated transgenic lines expressing cassava MeEXPA30. The vector pOGT was first digested with $X \mathrm{cml}$ restriction enzyme. The fragment of MeEXPA30 was amplified from cassava genome followed by the addition of adenosine overhangs at the $3^{\prime}$ end of the PCR products. Thus, the PCR fragments could be directly cloned into $X c m$-digested pOGT vector through TA cloning (Figure 3A). The construct of pOGTMeEXPA30 was transformed into Arabidopsis, and a similar screening process as for the plants transformed with POGT was performed. Fluorescence detection showed that nearly $0.5 \%$ of $T_{1}$ seeds and $28 \%$ of $T_{2}$ seeds had fluorescence (Figure $3 \mathrm{~B}-\mathrm{F}$ ). We also examined the amplification of MeEXPA30 by PCR analysis using genomic DNA from $T_{1}$ plants. Ten primary individual plants had showed the correct amplification, confirming transformation. These results demonstrate that the POGT vector is an effective system to generate transgenic Arabidopsis lines expressing foreign genes.

In recent years, fluorescent proteins have been widely used in screening transgenic seeds and plants [12-14]. Unlike methods using 4-methylumbelliferyl- $\beta$ D-glucopyranoside or Pdf2.1::DSRED as a selection marker, the pOGT system does not require pretreatments to induce the expression of fluorescent proteins. Although OLE1-GFP has also been previously used as a screening marker in the fluorescenceaccumulating seed technology (FAST) method [5], the FAST method uses the gateway cloning system to introduce the foreign genes. This method contains two steps: cloning the DNA fragment into a general donor plasmid and transferring into expression vectors [5]. Such two-step cloning and expensive reagents limit the gateway system to use for large-scale cloning [6]. The pOGT system we developed in this study utilizes a one-step TA cloning strategy for engineering the expression construct, simplifying the cloning method.

\section{FUTURE PERSPECTIVE}

The Agrobacterium-mediated floral-dip method was originally used for generating transgenic Arabidopsis thaliana. In recent years, it has also been used for other plants such as wheat [15]. Therefore, the principle of pOGT may also be applied to other oil-rich plants, giving the pOGT system broader impact.

\section{SUPPLEMENTARY DATA}

To view the supplementary data that accompany this paper please visit the journal website at: www.future-science. com/doi/suppl/10.2144/btn-2019-0141

\section{AUTHOR CONTRIBUTIONS}

L-J Luo, Q Liu, L-Y Jiang and M Li designed the research. $M$ Li and Q Liu performed the experiments. M Li, Q Liu, L-Y Jiang, Y-H Wu, L-J Luo, Y-H Chen and L-J Luo analyzed the data. L-Y Jiang and M Li wrote the paper. All authors contributed to writing this manuscript and approved of its final version.

\section{FINANCIAL \& COMPETING INTERESTS DISCLOSURE}

This work was supported by grants from the National Natural Science Foundation of China (31660412), the Integrated demonstration of key techniques for the industrial development of featured crops in rocky desertification areas of Yunnan-GuangxiGuizhou provinces (SMH2019-2021), the National key research and development program (2018YFD0201109-5) and the Hainan University Startup Fund (KYQD(ZR)1912). The authors have no other relevant affiliations or financial involvement with any organization or entity with a financial interest in or financial conflict with the subject matter or materials discussed in the manuscript apart from those disclosed.

No writing assistance was utilized in the production of this manuscript.

\section{OPEN ACCESS}

This work is licensed under the AttributionNonCommercial-NoDerivatives 4.0 Unported License. To view a copy of this license, visit http://creativecommons.org/licenses/ by-nc-nd/4.0/

\section{REFERENCES}

Papers of special note have been highlighted as: $\bullet$ of interest

1. Rong WX, Hong S, Long YX. Studies on indoor multiplication of Arabidopsis thaliana seeds. J. Cent. South Univ. 23(3), 91-94 (2002).

2. Juan $\mathrm{H}$, Yang LJ. Arabidopsis genome sequencing and plant functional genomics. Microbiology 28(3), 99-101 (2001).

3. Yin $\mathrm{XL}$, Jiang $\mathrm{L}$, Song $\mathrm{NH}$, Yang $\mathrm{H}$. Toxic reactivity of wheat (Triticum aestivum) plants to herbicide isoproturon. J. Agric. Food Chem. 56(12), 4825-4831 (2008).

4. Shimada TL, Shimada T, Takahashi H, Fukao Y, Hara-Nishimura I. A novel role for oleosins in freezing tolerance of oilseeds in Arabidopsis thaliana. Plant J. 55(5), 798-809 (2008).

5. Shimada TL, Shimada T, Hara-Nishimura I. A rapid and non-destructive screenable marker, FAST, for identifying transformed seeds of Arabidopsis thaliana. Plant J. 61(3), 519-528 (2010).

- A new time-saving method for screening transgenic $A r a b i$ dopsis thaliana.

6. Chen $\mathrm{S}$, Songkumarn $\mathrm{P}, \mathrm{Liu} \mathrm{J}$, Wang $\mathrm{GL}$. A versatile zero background T-vector system for gene cloning and functional genomics. Plant Physiol. 150(3), 1111-1121 (2009).

Development of a T-vector, providing a general, cost-efficient and high-throughput platform for gene function study.

7. Huala E, Dickerman AW, Garcia-Hernandez M et al. The Arabidopsis Information Resource (TAIR): a comprehensive database and web-based information retrieval, analysis, and visualization system for a model plant. Nucleic Acids Res. 29(1), 102-105 (2001).

- Development of The Arabidopsis Information Resource (http://www.Arabidopsis.org), often used in Arabidopsis

8. Clarke JD. Cetyltrimethyl ammonium bromide (CTAB) DNA miniprep for plant DNA isolation. Cold Spring Harb. Protoc. 2009(3), pdb.prot5177 (2009).

Simple, fast method for extracting plant genomes, since refined and now widely used by many researchers.

9. Clough SJ, Bent AF. Floral dip: a simplified method for agrobacterium mediated transformation of Arabidopsis thaliana Plant J. 16(6), 735-743 (1998)

- A successful method for transformation of Arabidopsis, which has greatly facilitated the research of gene function.

10. Xu L, Liu C, Chang Y. Theory and method of double-standard curves method of relative quantification PCR. Biotechnol. Bull. 1, 70-75 (2011).

11. An YQ, Mcdowell JM, Huang S, Chambliss S, Mckinney EC, Meagher RB. Strong, constitutive expression of the Arabidopsis ACT2/ACT8 actin subclass in vegetative tissues. Plant J. 10(1), 107-121 (1996).

12. Wei $S, B r a v d o B$, Shoseyov $O$. Fluorescent screening of transgenic Arabidopsis seeds without germination. Plant Physiol. 135(2), 709-714 (2004).

13. Ali MA, Shah KH, Bohlmann H. pMAA-Red: a new pPZP-derived vector for fast visual screening of transgenic Arabidopsis plants at the seed stage. BMC Biotechnol. 12, 37 (2012).

14. Stuitje AR, Verbree EC, Van der Linden KH, Mietkiewska EM, Nap JP, Kneppers TJ. Seed-expressed fluorescent proteins as versatile tools for easy (co)transformation and hroins as Biotechnol. J. 1(4), 301-309 (2003).

15. Agarwal S, Loar S, Steber C, Zale J. Floral transformation of wheat. Methods Mol. Biol. 478(1), 105-113 (2009). 